\title{
Carga de cuidado de los cuidadores familiares y nivel de dependencia de su familiar*
}

\author{
Burden of care of family caregivers and level of dependency of their family member \\ Carga de cuidado dos cuidadores familiares e nível de dependência do parente
}

\author{
Angela María Salazar Maya ${ }^{\mathrm{a}}$ \\ Universidad de Antioquia, Colombia \\ angela.salazar@udea.edu.co \\ ORCID: https://orcid.org/0000-0001-7599-1193 \\ Yadira Cardozo García \\ Universidad de Antioquia, Colombia \\ ORCID: https://orcid.org/0000-0003-2588-5717 \\ Carmen Liliana Escobar Ciro \\ Universidad de Antioquia, Colombia \\ ORCID: https://orcid.org/0000-0002-6296-0280
}

Aceptado: 25 Mayo 2020

Publicado: 22 Julio 2020

DOI: https://doi.org/10.11144/Javeriana.ie22.cccf

\section{Resumen:}

Introducción: La enfermedad crónica, los procesos terminales, la vejez y la supervivencia de personas con comorbilidades, discapacidades físicas o psíquicas, además de los cambios en los sistemas de salud, han trasladado el cuidado a la familia y a los cuidadores familiares. Objetivo: Determinar la carga del cuidado en el cuidador familiar y el nivel de dependencia funcional o enfermedad crónica de su familiar. Método: Estudio cuantitativo, descriptivo de corte trasversal, desarrollado en la ciudad de Medellín (Colombia) de noviembre de 2017 a septiembre de 2019. La muestra fueron 494 personas, a conveniencia. Se aplicaron los instrumentos Zarit, Pulses y variables sociodemográficas. Los datos se analizaron con el programa SPSS versión 25. A las variables de tipo cuantitativo se les aplicaron medidas de centro y dispersión. Para las cualitativas se analizaron sus frecuencias. Resultados: El 89,7 \% de la muestra son del sexo femenino, con una edad media de 53,5 años; el 39\% está soltero; el 75,6\% estudió hasta secundaria; el 83,1 \% se dedica al hogar y lleva como cuidador 8,6 años, y el 87,9\% es el cuidador principal, con problemas del sistema cardiovascular, nervioso y osteomuscular. Para el 29,4\% de los participantes la carga es severa, seguida de moderada y el nivel de dependencia de la persona que cuidan es severo, en el 71 \% de los casos. Conclusiones: En nuestro medio, la mayoría son cuidadoras, solteras, encargadas del hogar y cuidan a un familiar (padre-hijo). La sobrecarga del cuidado es excesiva, porque cuidan a personas con dependencia severa.

Palabras clave: cuidadores, enfermedad crónica, dependencia, cuidado en custodia.

\section{Abstract:}

Introduction: Changes in healthcare systems, in addition to chronic illness, terminal processes, old age, and survival of people with comorbidities, physical or mental disabilities, have transferred care to families and family caregivers. Objective: To determine the burden of care on family caregivers and the level of functional dependency or chronic illness of their family member. Method: Quantitative, descriptive cross-sectional study, carried out in the city of Medellin (Colombia) from November 2017 to September 2019. The sample was comprised of 494 people, as appropriate. The Zarit and Pulses instruments were applied, and sociodemographic variables were taken into account. Data were analyzed with the SPSS version 25 program. Measures of centrality and dispersion were applied to the quantitative variables. Frequencies were analyzed for qualitative variables. Results: $89.7 \%$ of the sample are female, with a mean age of 53.5 years; $39 \%$ are single; $75.6 \%$ studied until high school; $83.1 \%$ are in charge of household tasks and have been a caregiver for 8.6 years, and $87.9 \%$ are the main caregiver, with problems of the cardiovascular, nervous, and musculoskeletal systems. For $29.4 \%$ of the participants, the burden is severe, followed by moderate, and the level of dependency of the person they care for is severe in $71 \%$ of the cases. Conclusions: In our environment, the majority of caregivers are single women, housekeepers, and take care of a relative (father, son).

The overload of care is excessive because they care for people with severe dependency.

Keywords: caregivers, chronic illness, dependence, custodial care.

Notas de autor

a Autora de correspondencia. Correo electrónico: angela.salazar@udea.edu.co 


\section{Resumo:}

Introdução: Doenças crônicas, processos terminais, velhice e sobrevivência de pessoas com comorbilidades, deAciências físicas ou psíquicas, além de alteraçóes nos sistemas de saúde, trasladaram o cuidado para a família e cuidadores familiares. Objetivo: Determinar a carga de cuidado no cuidador familiar e o nível de dependência funcional ou doença crônica do familiar. Método: Estudo quantitativo, descritivo de corte transversal, realizado na cidade de Medellín (Colômbia) de novembro de 2017 a setembro de 2019. A amostra foi de 494 pessoas aleatórias. Os instrumentos Zarit, Pulses e variáveis sócio-demográficas foram aplicados. Os dados foram analisados com o programa SPSS versão 25. Medidas de centro e dispersão foram aplicadas às variáveis de tipo quantitativo. Para as qualitativas analisaram-se as frequências. Resultados: 89,7\% da amostra são de sexo feminino, com idade média de 53,5 anos; 39 \% são solteiros; $75,6 \%$ estudaram até secundário; 83,1\% são donos da casa e cuidam há 8,6 anos, e 87,9\% são principais cuidadores, com problemas de sistema cardiovascular, nervoso e musculoesquelético. Para 29,4\% dos participantes, o ônus é grave, seguido de moderado e o nível de dependência da pessoa que cuida é grave, em 71 \% dos casos. Conclusões: No nosso meio, a maioria é cuidadora, solteiras, dona da casa e cuida de parente (pai-Alho). A sobrecarga do cuidado é excessiva porque elas cuidam de pessoas com dependência grave.

Palavras-chave: cuidadores, doença crônica, dependência, cuidado em custodia.

\section{Introducción}

La población de Medellín (Colombia), en el 2017, fue de 2.508.500, que corresponde al 5,112 \% del total de la población colombiana. Si el aumento de la población fuera igual al periodo $2015-2017$ (+0,89\% por año), para el 2019 se esperaban 2.553.372 habitantes (1). Con una esperanza de vida que pasó de 75,2 años en el quinquenio 2006-2010 a 78,2 años para el periodo 2016-2017 (2).

Por otro lado, durante el 2017 en la ciudad se realizaron 5.829 .429 consultas, el $11 \%$ de estas correspondió a la hipertensión esencial, siendo la primera causa de atención. Le siguen "otros síntomas y hallazgos anormales clínicos y de laboratorio, no clasificados” con 407.592 consultas; la diabetes mellitus, con 227.282, y en último lugar otras infecciones agudas del sistema respiratorio superior, con 216.772 (2).

Las enfermedades cardiacas, en especial las isquémicas, siguen ocupando el primer lugar entre las causas de mortalidad desde el 2012 (14,5\%); en segundo lugar, desde el 2013 están las enfermedades crónicas del sistema respiratorio inferior $(7,9 \%)$, seguidas de las cerebrovasculares $(6,4 \%)$, las hipertensivas $(5,6 \%)$ y la neumonía $(4,6 \%)(2)$.

Por otra parte, el Boletín 9 del Observatorio Nacional de Discapacidad (3) dio a conocer un estudio ecológico-exploratorio que caracteriza a las personas con discapacidad en cinco ciudades de Colombia: Cartagena, Bogotá, Medellín, Cali y Barranquilla. Los adultos mayores se encuentran en el grupo con la mayor prevalencia de discapacidad entre la población: muchas de ellas dificultades de movilidad y la mayoría de los participantes con bajo nivel educativo, lo que puede generar implicaciones económicas, sociales, Asiológicas para el cuidador, la familia y su cuidado.

Es importante resaltar que más personas de todas las edades viven con enfermedades crónicas como las cardiovasculares, la diabetes, la artritis y alteraciones de salud mental (3). Así mismo, el envejecimiento de la población crea más demanda en los servicios de salud, y el cuidado principal es asumido por la familia. Los cuidadores familiares (4) se definen como:

[...] la persona que, siendo cónyuge, compañero o compañera permanente, pariente hasta el quinto grado de consanguinidad, tercero de aAnidad, primero civil o que, sin tener ningún tipo de parentesco con la persona con dependencia de cuidado, es apoyo permanente para las actividades básicas e instrumentales de la vida diaria, sin recibir contraprestación económica por su asistencia y que por su labor dedicada al cuidado de otra persona. (5)

Cuidar a un familiar enfermo implica ciertas restricciones, como aislarse, disminuir sus actividades sociales, descuidar su propia salud, aumentar su carga de trabajo, disminuir o perder los ingresos económicos, producir cambios en la relación con el paciente o generar problemas sexuales (6). La literatura describe que si el cuidado se mantiene durante un largo período, puede desencadenar problemas de salud en el cuidador, que van desde fatiga hasta problemas emocionales, como depresión, ansiedad, entre muchos otros (7-9). 
A su vez, la dependencia de una persona se presenta cuando hay disfuncionalidad y discapacidad que requiere la ayuda de otro para llevar a cabo actividades de la vida diaria, como desplazarse o alimentarse. Esto tiene implicaciones psicológicas tanto para la persona que la padece como para la familia. Otras que se pueden presentar son las de tipo social, político y económico. Lo anterior se constituye en un estresor con efectos negativos en la persona que cuida, y si está sola en la labor de cuidado, probablemente presentará carga o sobrecarga (10).

Por asuntos como los relatados, un grupo de docentes investigadores de la Facultad de Enfermería de la Universidad de Antioquia decidió en el 2006, y motivadas por los resultados de investigaciones como: "La transición del cuidado del hospital a la casa de ancianos dependientes" (11), "Impacto en la biografía de los cuidadores de pacientes con demencia" $(12,13)$, "Cuidadores familiares de ancianos" $(14,15)$ o "El cuidado artesanal la invención ante la adversidad" (16), elaborar un programa de extensión solidaria en alianza con la Pastoral de la Salud de la Arquidiócesis de Medellín, dirigido a cuidadores familiares de personas en situación de enfermedad o personas mayores con algún grado de dependencia funcional. El objetivo del programa aún vigente es "Capacitar a cuidadores familiares y agentes de pastoral de la salud, en elementos básicos para el cuidado en el hogar de personas en situación de enfermedad, con el an de contribuir al bienestar tanto de las personas cuidadas como de quienes proveen el cuidado" (17). En dicho programa se han capacitado más de 529 personas y lleva 12 años de trabajo ininterrumpido.

Además, en el 2014, la Facultad de Enfermería de la Universidad de Antioquia participó activamente en la elaboración de una política pública para los cuidadores del Municipio de Medellín. Esta iniciativa dio lugar al Acuerdo 27 de 2015 (5): "Por medio del cual se establece una política pública para cuidadoras y cuidadores familiares y voluntarios de personas con dependencia de cuidado y conformación de Redes Barriales de Cuidado en el Municipio de Medellín", cuyo propósito es:

Reconocer a la persona cuidadora como eje fundamental en el cuidado y bienestar de personas dependientes de cuidado, reconociéndoles como sujeto de derechos y también de cuidado lo cual les debe permitir acceder y ser atendidas de manera preferencial y oportuna a los programas de desarrollo, promoción y prevención para el control de riesgos generales y especíacos derivados del cuidado permanente a otra persona. (5)

Así mismo, la Alcaldía de Medellín creó el Observatorio de Envejecimiento y Vejez de la ciudad, el cual hace seguimiento a la política pública (18). La Alcaldía de Medellín, a través de Amautta (la Unidad de Personas Mayores, adscrita a la Subsecretaría de Grupos Poblacionales de la Secretaría de Inclusión Social y Familia) y la unidad de discapacidad, han capacitado a los cuidadores familiares de las diferentes comunas de la ciudad (18).

Ante el incremento del número de cuidadores familiares en la ciudad y con los dos programas de capacitación descritos, se consideró importante determinar la carga del cuidado en el cuidador familiar y el nivel de dependencia funcional o enfermedad crónica de su familiar.

\section{Método}

Estudio descriptivo-trasversal (19) en dos programas de capacitación de cuidadores familiares de la ciudad de Medellín, entre noviembre del 2017 y septiembre del 2019. En el estudio participaron 496 cuidadores. La muestra fue obtenida a conveniencia.

Los criterios de inclusión fueron los cuidadores mayores de edad que participaron en la capacitación de ambas entidades: Facultad de Enfermería de la Universidad de Antioquia (26,76\%) y Alcaldía de Medellín (73,24\%). Los participantes fueron contactados por las investigadoras en el momento de la capacitación o por vía telefónica. Se les informó acerca de los objetivos del estudio y después de Armar el consentimiento informado, los participantes respondieron a las preguntas del cuestionario ad hoc de variables sociodemográficas y las preguntas de los instrumentos Pulses y Zarit Burden Inventori. 
La escala Pulses es un instrumento de 6 ítems, adaptado por Granger et al. (20). Mide la dependencia o funcionalidad de la persona cuidada y la clasiaca con dependencia leve (6-8 puntos), moderada (9-10 puntos) o severa (11-24 puntos), según la estabilidad de la patología o condición física, el uso de extremidades superiores, la marcha o movilidad, el uso de los miembros inferiores, la función sensorial, el control de los esfínteres y la capacidad de socializar. Cada función se caliaca de 1 a 4 . Si es independiente, 1; si requiere apoyo (mecánico o aparato), 2; si requiere apoyo mecánico o de otra persona, 3, y si está dependiente, 4. El alfa de Cronbach obtenido en nuestro estudio fue de 0,790.

Por otra parte, el Zarit Burden Inventory (21) mide la carga del cuidador. Consta de 22 ítems y 5 opciones de respuesta que van desde $0=$ nunca, $1=$ rara vez, $2=$ algunas veces, $3=$ bastantes veces y $4=$ casi siempre. Permite clasiacar la carga en: "carga nula", si la sumatoria es menos de 23 puntos; "carga leve", entre 24 y 30 puntos; "carga moderada", entre 31 y 40 puntos, y "carga severa”, más de 41 . En el presente estudio la escala obtuvo una conAabilidad medida por alfa de Cronbach de 0,863 .

Se consolidó una base de datos en Excel, revisada y depurada buscando inconsistencias. Posteriormente, fue exportada al programa SPSS versión 25 para describir la carga de cuidado en el análisis estadístico. Mediante el cálculo de medias y desviación estándar se analizaron las variables que miden los instrumentos de recolección. Luego se reclasiacaron las variables según la puntuación obtenida, con las cuales se realizó un análisis de frecuencias absolutas y relativas. Se exploraron posibles relaciones entre las variables sociodemográAcas y la puntuación obtenida con el Zarit y el Pulses, usando pruebas chi-cuadrado $\left(\chi^{2}\right.$.).

Las investigadoras aplicaron el instrumento, con el cual habían entrenado previamente y del cual habían uniacado criterios, con el an de minimizar los sesgos. Los cuidadores participaron de forma voluntaria, previa información de los objetivos, los aspectos relacionados con la custodia de la información, sus Anes, la devolución de resultados y la conAdencialidad, previa a la Arma del consentimiento informado. Se siguieron todas las recomendaciones dadas por la Resolución 8430 de 1993 (22) y los lineamientos éticos internacionales de la investigación biomédica en seres humanos, establecidas por el Consejo de las Organizaciones Internacionales de Ciencias Médicas, en colaboración con la Organización Mundial de la Salud (23). Se obtuvo el aval del Comité de Ética de Investigación de la Facultad de Enfermería de la Universidad de Antioquia, Acta CEI-FE 2017-46. Igualmente, se obtuvo el permiso para el uso del instrumento Zarit.

\section{Resultados}

\section{Características sociodemográficas}

En la tabla 1 se presentan las características sociodemográAcas relacionadas con la edad, el sexo, el estado civil, la escolaridad, la ocupación y la residencia de los participantes.

TABLA 1.

Características sociodemográAcas

\begin{tabular}{llrr}
\hline Variables sociodemográficas & n & \% \\
\hline Edad & $19-28$ & 15 & 3,0 \\
\cline { 2 - 4 } & $29-38$ & 28 & 5,7 \\
\cline { 2 - 4 } & $39-48$ & 92 & 18,7 \\
\cline { 2 - 4 } & $49-58$ & 193 & 39,2 \\
\cline { 2 - 4 } & $59-68$ & 136 & 27,6 \\
\cline { 2 - 4 } & $69-78$ & 23 & 4,7 \\
\cline { 2 - 4 } & $79-88$ & 5 & 1,0 \\
\cline { 2 - 4 } Sexo & Total & 492 & 100,0 \\
\hline Hombre & 51 & 10,3 \\
\hline Estado civil & Mujer & 443 & 89,7 \\
\cline { 2 - 4 } & Total & 494 & 100,0 \\
\cline { 2 - 4 } & Soltero & 198 & 40,2 \\
\cline { 2 - 4 } & Casado & 136 & 27,6 \\
\cline { 2 - 4 } & Separado & 67,6 \\
\cline { 2 - 4 } & Viudo & 34 & 6,9 \\
\cline { 2 - 4 } & Unión libre & 58 & 11,8 \\
\cline { 2 - 4 } & Total & 493 & 100,0
\end{tabular}

\begin{tabular}{llrr}
\hline Variables sociodemográficas & n & $\%$ \\
\hline Escolaridad & $\begin{array}{l}\text { Primaria } \\
\text { incompleta }\end{array}$ & 73 & 14,9 \\
\cline { 2 - 4 } & $\begin{array}{l}\text { Primaria } \\
\text { completa }\end{array}$ & 70 & 14,3 \\
\cline { 2 - 4 } & $\begin{array}{l}\text { Bachillerato } \\
\text { incomplero }\end{array}$ & 88 & 18,0 \\
\cline { 2 - 4 } & $\begin{array}{l}\text { Bachillerato } \\
\text { completo }\end{array}$ & 139 & 28,4 \\
\cline { 2 - 4 } & $\begin{array}{l}\text { Técnico } \\
\text { tecnológico }\end{array}$ & 72 & 14,7 \\
\cline { 2 - 4 } & $\begin{array}{l}\text { Universitario } \\
\text { incompleto }\end{array}$ & 13 & 2,7 \\
\cline { 2 - 4 } & $\begin{array}{l}\text { Universitario } \\
\text { completo }\end{array}$ & 31 & 6,3 \\
\cline { 2 - 4 } & Posgrado & 3 & 0,6 \\
\cline { 2 - 4 } & Total & 489 & 100,0 \\
\hline & & & \\
\hline
\end{tabular}

\begin{tabular}{llrr}
\hline Variables sociodemográficas & $\mathbf{n}$ & $\mathbf{\%}$ \\
\hline Ocupación & Hogar & 412 & 83,1 \\
\cline { 2 - 4 } & Empleado & 9 & 1,8 \\
\cline { 2 - 4 } & $\begin{array}{l}\text { Trabajo } \\
\text { independiente }\end{array}$ & 55 & 11,1 \\
\cline { 2 - 4 } & Estudiante & 4 & 0,8 \\
\cline { 2 - 4 } & Otro & 16 & 3,2 \\
\cline { 2 - 4 } & Total & 496 & 100,0 \\
\hline Lugar de residencia & Medellín & 493 & 99,4 \\
\cline { 2 - 4 } & $\begin{array}{l}\text { Área } \\
\text { metropolitana }\end{array}$ & 3 & 0,6 \\
\cline { 2 - 4 } & Total & 496 & 100,0 \\
\hline
\end{tabular}


La edad promedio fue de 53,5 años y en la tabla 1 se destaca una mayor prevalencia en participantes pertenecientes a la década de los 50, la mayoría bachilleres, solteras y dedicadas al hogar.

En la tabla 2 se relacionan las variables sociodemográAcas con las escalas de Pulses y Zarit, recodiAcadas en carga y dependencia severa y sin carga severa (que corresponde a carga nula, leve moderada para el Zarit) y sin dependencia severa (que corresponde a dependencia leve y moderada para el pulses).

TABLA 2

Relación entre variables sociodemográAcas, el Pulses y el Zarit

\begin{tabular}{|c|c|c|c|c|c|c|c|c|c|c|c|}
\hline \multirow{3}{*}{ Variable } & & \multicolumn{4}{|c|}{ Pulses } & \multirow{3}{*}{$\mathbf{P}$} & \multicolumn{4}{|c|}{ Zarit } & \multirow{3}{*}{$\mathbf{P}$} \\
\hline & & \multicolumn{2}{|c|}{$\begin{array}{c}\text { Sin } \\
\text { dependencia } \\
\text { severa (leve } \\
\text { y moderada) }\end{array}$} & \multicolumn{2}{|c|}{$\begin{array}{c}\text { Dependencia } \\
\text { severa }\end{array}$} & & \multicolumn{2}{|c|}{$\begin{array}{c}\text { Sin carga } \\
\text { severa (de } \\
\text { nula a } \\
\text { moderada) }\end{array}$} & \multicolumn{2}{|c|}{$\begin{array}{l}\text { Carga } \\
\text { severa }\end{array}$} & \\
\hline & & $\mathbf{n}$ & $\%$ & $\mathbf{n}$ & $\%$ & & $\mathbf{n}$ & $\%$ & $\mathbf{n}$ & $\%$ & \\
\hline \multirow{7}{*}{ Edad } & $19-28$ & 1 & 0,7 & 14 & 4 & \multirow{7}{*}{$0,042^{*}$} & 11 & 3,2 & 4 & 2,8 & \multirow{7}{*}{0,883} \\
\hline & $29-38$ & 6 & 4,3 & 22 & 6,3 & & 19 & 5,5 & 9 & 6,2 & \\
\hline & $39-48$ & 27 & 19,1 & 65 & 18,6 & & 65 & 18,7 & 27 & 18,6 & \\
\hline & $49-58$ & 54 & 38,3 & 137 & 39,3 & & 137 & 39,5 & 56 & 38,6 & \\
\hline & $59-68$ & 40 & 28,4 & 96 & 27,5 & & 98 & 28,2 & 38 & 26,2 & \\
\hline & $69-78$ & 10 & 7,1 & 13 & 3,7 & & 14 & 4 & 9 & 6,2 & \\
\hline & $79-88$ & 3 & 2,1 & 2 & 0,6 & & 3 & 0,9 & 2 & 1,4 & \\
\hline \multirow{2}{*}{ Sexo } & Hombre & 10 & 7 & 41 & 11,7 & \multirow{2}{*}{0,209} & 39 & 11,2 & 12 & 8,3 & \multirow{2}{*}{62} \\
\hline & Mujer & 132 & 93 & 309 & 88,3 & & 310 & 88,8 & 133 & 91,7 & \\
\hline \multirow{5}{*}{ Estadocivil } & Soltero & 50 & 35,5 & 148 & 42,3 & \multirow{5}{*}{0,161} & 141 & 40,5 & 57 & 39,3 & \multirow{5}{*}{0,144} \\
\hline & Casado & 46 & 32,6 & 90 & 25,7 & & 87 & 25 & 49 & 33,8 & \\
\hline & Separado & 24 & 17 & 41 & 11,7 & & 54 & 15,5 & 13 & 9 & \\
\hline & Viudo & 7 & 5 & 27 & 7,7 & & 27 & 7,8 & 7 & 4,8 & \\
\hline & Unión libre & 14 & 9,9 & 44 & 12,6 & & 39 & 11,2 & 19 & 13,1 & \\
\hline \multirow{8}{*}{ Escolaridad } & Primaria incompleta & 26 & 18,4 & 47 & 13,6 & \multirow{8}{*}{0,091} & 49 & 14,1 & 24 & 16,9 & \multirow{8}{*}{0,288} \\
\hline & Primaria completa & 20 & 14,2 & 50 & 14,5 & & 48 & 13,8 & 22 & 15,5 & \\
\hline & Bachillerato incompleto & 18 & 12,8 & 69 & 19,9 & & 57 & 16,4 & 31 & 21,8 & \\
\hline & Bachillerato completo & 37 & 26,2 & 101 & 29,2 & & 104 & 30 & 35 & 24,6 & \\
\hline & Técnico tecnológico & 20 & 14,2 & 52 & 15 & & 52 & 15 & 20 & 14,1 & \\
\hline & Universitario incompleto & 5 & 3,5 & 8 & 2,3 & & 10 & 2,9 & 3 & 2,1 & \\
\hline & Universitario completo & 12 & 8,5 & 19 & 5,5 & & 25 & 7,2 & 6 & 4,2 & \\
\hline & Posgrado & 3 & 2,1 & 0 & 0 & & 2 & 0,6 & 1 & 0,7 & \\
\hline \multirow{5}{*}{ Ocupación } & Hogar & 119 & 83,8 & 291 & 82,7 & \multirow{5}{*}{0,063} & 290 & 82,9 & 122 & 83,6 & \multirow{5}{*}{0,663} \\
\hline & Empleado & 2 & 1,4 & 7 & 2 & & 7 & 2 & 2 & 1,4 & \\
\hline & Trabajo independiente & 13 & 9,2 & 42 & 11,9 & & 39 & 11,1 & 16 & 11 & \\
\hline & Estudiante & 3 & 2,1 & 1 & 0,3 & & 3 & 0,9 & 1 & 0,7 & \\
\hline & Otro & 5 & 3,5 & 11 & 3,1 & & 11 & 3,1 & 5 & 3,4 & \\
\hline \multirow{2}{*}{ Residencia } & Urbana & 134 & 94,4 & 329 & 93,5 & \multirow{2}{*}{0,606} & 324 & 92,6 & 141 & 96,6 & \\
\hline & Rural & 8 & 5,6 & 23 & 6,5 & & 26 & 7,4 & 5 & 3,4 & 0,337 \\
\hline
\end{tabular}

* Estadísticamente signiAcativo. El valor de p es menor a 0,05 para una prueba de chi-cuadrado. Fuente: elaboración propia

$\mathrm{Al}$ analizar la relación entre las variables sociodemográficas y la carga severa o la dependencia severa, se observa una significancia estadística entre la edad del cuidador y la dependencia severa de la persona cuidada. En la muestra, el 39,3 \% de los que refieren una dependencia severa tiene un cuidador con edad entre 49 y 58 años. Igualmente, prevalecen las cuidadoras mujeres, solteras, bachilleres, dedicadas al hogar, a cargo de personas con dependencia severa. Sin embargo, no existe suAciente evidencia estadística en los datos para afirmar que dichas variables estén asociadas con la puntuación del Pulses $(p>0,05)$, como se observa en la tabla 2.

$\mathrm{Al}$ comparar el instrumento Zarit con los datos sociodemográficos, se encontró que pese a no haber asociación estadísticamente significativa $(p>0,05)$, se observa que el 39,5\% de los participantes está en el rango de edad entre 49 y 58 años, y para estos el ser cuidador supone una carga severa en el 38,6\% de los participantes. Acerca del estado civil, el porcentaje que prevalece es el de los solteros, donde el 40,5\% no asume una carga severa, a diferencia de los casados, quienes perciben en un 33,8 \% carga severa del cuidado.

El $30 \%$ de los cuidadores tienen bachillerato completo y se aprecian sin carga severa, y el 24,6\%, con carga severa.

En cuanto a la ocupación, el 82,9\% de la muestra que se dedican a las labores del hogar se perciben sin carga severa, y el 83,60 \% dedicado a las mismas labores se perciben con una carga severa. En tabla 3 observamos el cruce de las puntuaciones entre el Zarit y el Pulses. Resalta el hecho de que el 24,5\% percibe una carga severa; el $2 \%$, carga moderada, y el $3 \%$, carga leve. 
TABLA 3.

Relación entre Zarit y Pulses

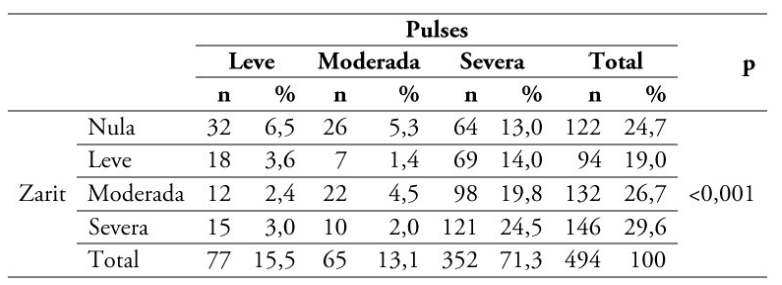

Fuente: elaboración propia

\section{Carga de cuidado de los cuidadores}

Respecto a las categorías de las puntuaciones, existe asociación estadísticamente signiacativa entre lo obtenido con el Zarit y el Pulses $(p<0,05)$. Llama la atención que el 24,5\% percibe una carga severa en ambas escalas; el 2 \%, moderada en Pulses, pero severa en Zarit y un 3 \% carga leve en Pulses pero severa en Zarit como se observa en la tabla 3.

Al revisar el Zarit por ítem, en nuestro estudio se resalta que no sienten vergüenza por el comportamiento del familiar enfermo (78,1\%); nunca se sienten enojados con su familiar enfermo $(53,9 \%)$; tampoco sienten que la situación de su familiar afecta de manera negativa la relación con sus familiares y amigos (52,32\%); no sienten que cuidar a su familiar afecta su estado de salud (41,21\%); no desean que su familiar sea cuidado por personas diferentes $(53,53 \%)$, y no se sienten inseguros en qué hacer con el familiar $(56,56 \%)$.

El 43,43 \% de los cuidadores manifestó temor por el futuro de su familiar. El 42,2 \% percibe que el paciente espera de su cuidador atención, como si fuera la única persona con la que pudiera cuidar. El $61,61 \%$ de los cuidadores considera que tenían la capacidad de dedicarse al cuidado de su familiar por mucho más tiempo.

\section{Nivel de dependencia}

Medido por el Pulses, se observa que el 71,3 \% de las personas cuidadas tiene un nivel de dependencia severo; seguido del $15,5 \%$, con una dependencia leve (tabla 4 ).

TABLA 4.

Media, desviación estándar y cuartiles. Puntuación Zarit-Pulses

\begin{tabular}{lccc}
\hline Concepto & Zarit & Pulses \\
Media & & 33,9113 & 13,82 \\
Desviación estándar & 14,62239 & 4,751 \\
& 25 & 24,0000 & 10,00 \\
Percentiles & 50 & 33,0000 & 14,00 \\
& 75 & 43,7500 & 17,00 \\
\hline
\end{tabular}

Fuente: datos investigación

Se puede ultimar que la media del Zarit dentro de la muestra fue del 33,91, lo que enseña una carga moderada y una media del pulses de 13,82 describe un nivel de dependencia severo. De la muestra con relación al Zarit, el $25 \%$ de la muestra obtuvo un puntaje inferior o igual a 24; el $50 \%$ obtuvo 33 o menos y el 75 \%obtuvo una puntuación igual o inferior de 43,7. Con relación al Pulses el $25 \%$ obtuvo un puntaje inferior o igual a 10; el $50 \%$ igual o inferior a 14; y el $75 \%$ igual o inferior a 17 . Como se observa en la tabla 
4; por tanto, el $50 \%$ de los participantes se encuentran entre carga moderada y severa. Igualmente, la persona que cuida está en un nivel de dependencia moderado o severo.

\section{Discusin}

En el contexto de la ciudad de Medellín poco se ha estudiado a los cuidadores familiares, aunque si se han capacitado. El presente estudio nos mostró las características de algunos de los cuidadores de la ciudad, su carga de cuidado y el nivel de dependencia de las personas que cuidan.

$\mathrm{Al}$ igual que otros estudios, como los reportados por Coca et al. (24), Sánchez et al. (25), Hanzeliková et al. (26) y Pascual et al. (27), las características sociodemográficas de los cuidadores son similares, en cuanto al género, el estado civil, la ocupación y el estrato socioeconómico. Continúan siendo mujeres en su gran mayoría, probablemente por lo que expresan Jiménez Ruiz y Moya Nicolás (28), el cuidado en la mujer es comprendido como algo natural, y una obligación moral dentro de la familia. Además, el mismo autor señala que en la familia puede presentarse una distribución de actividades para el cuidado que depende del género: si es una mujer, puede encargarse del cuidado total; pero si es un hombre, probablemente, realizará tareas como: "echar un ojo".

Con relación a la edad de los cuidadores, se evidencia que estos son adultos medios en un porcentaje alto, como lo encontrado por Hanzeliková et al. (26), Pascual et al. (27), lo cual podría entenderse para nuestro estudio, debido a que Medellín es una de las ciudades con mayor número de personas ancianas y cuyas familias, desde lo cultural, asumen el cuidado en el hogar (29). De igual forma, el cuidado recae en su mayoría en las personas solteras, por considerarse que tienen disponibilidad de tiempo para ejercer esta labor, como lo enseña nuestro estudio.

Los resultados evidencian que los cuidadores en la ciudad de Medellín tienen estudios secundarios y universitarios completos, similar a lo encontrado por Gómez-Galindo et al. (30). Para la ciudad de Medellín, con relación al resto del país, el índice de analfabetismo es el más bajo, y ello se convierte en una oportunidad para el apoyo y la capacitación de estos cuidadores. A esto se añade que en las investigaciones de autores como Hanzeliková et al. (26), Coca et al. (24), Pascual et al. (27), Piratoba-Hernández y Rozo-Gutiérrez (31) se documentó la existencia de una relación filial entre el cuidador y la persona cuidada. Cabe anotar que los cuidadores con mayor capacidad económica tienen la posibilidad de contratar a personas con formación para cuidar a su familiar en casa o llevarlo a una institución.

Así, también, los principales problemas de salud de las personas cuidadas y de los cuidadores son las relacionadas con la presencia de enfermedades crónicas. Prieto et al. (32), Sánchez et al. (25) y GómezGalindo et al. (30) encontraron resultados similares en sus investigaciones, en las cuales los cuidadores padecían enfermedades crónicas, situación que se explica si consideramos que dentro de los problemas cardiovasculares las primeras causas de consulta en la ciudad son la hipertensión y la diabetes mellitus, sumado al aumento mundial de estas enfermedades en la población adulta, ya sea por los estilos de vida de los cuidadores o por el componente genético de estas, al ser afecciones de larga duración conllevan la necesidad de un cuidador familiar.

En general, cuidar a una persona mayor o con enfermedad crónica se caracteriza porque no solo poseen una enfermedad, sino que prevalece la pluripatología, lo que los hace más vulnerables y frágiles; por lo tanto, requieren cuidados complejos. Son personas con patologías que cuidan a personas enfermas o dependientes.

Los resultados encontrados en el estudio muestran la carga de los cuidadores de la ciudad de Medellín y no se alejan de lo documentado en otras investigaciones, donde los cuidadores perciben sobrecarga. RamónArbuésa et al. (33) reportaron síndrome de sobrecarga en el $48 \%$ de la muestra; así mismo, Blanco et al. (34), para quienes el 55,4\% de la muestra presentó una media de 27,3 (DT = 13,3) en la carga del cuidado. 
El estudio de Prieto-Miranda et al. (32) indicó una sobrecarga: intensa (11,1\%), moderada (16,7\%) y sin sobrecarga $(72,22 \%)$.

Pinzón y Carrillo (35) describieron que el 23,7 \% de los participantes presentó sobrecarga severa; el 27,3 \% leve, y el $49 \%$, sin sobrecarga. Los anteriores son datos similares a los de Martínez Debs et al. (36), para quienes el $42 \%$ se ubicó en un rango de sobrecarga de leve a moderada y el $58 \%$ presentó sobrecarga de moderada a severa. No existieron cuidadores con niveles severos de sobrecarga. Además, el mismo estudio describió que el $87 \%$ de los cuidadores consideró que tenía la capacidad de dedicarse al cuidado de su familiar por mucho más tiempo, similar a lo encontrado en nuestro estudio. Por otro lado, los cuidadores del estudio expresaron su negativa de encargar el cuidado del familiar a otras personas, al tiempo que no predominaron sentimientos de enfado por tener que brindar el cuidado al paciente (36). Resultados similares al nuestro.

En un porcentaje elevado, el grado de dependencia de las personas cuidadas en nuestro estudio es de dependencia severa, y al compararlo con la literatura, se encontró que Cantillo-Medina et al. (37) reportan cifras similares, en cuanto al nivel funcional, siendo un alto nivel de dependencia 68,6\% el de las personas con enfermedad crónica. Contrario a esto, Sánchez Herrera et al. (38) indicaron que el 60,62 \% de las personas enfermas tenía baja dependencia; el 26,41\%, dependencia moderada, y el 12,97 \%, dependencia severa. La mayoría de los participantes cuida a personas con dependencia moderada, en un 42,6 \%; leve, en un $31,6 \%$; severa, en un 24,8 \%, y de un $1 \%$ no se obtuvo información. Torres-Pinto et al. (39) reportaron un nivel de dependencia funcional bajo.

Los resultados del estudio aportan un conocimiento local al municipio de Medellín y a la disciplina de enfermería, y en este se reconocieron las necesidades de los cuidadores, lo que nos lleva a repensar la práctica de educación para la salud, no solo para la persona que cuida, sino para sí mismos, una educación que permita la solución de problemas, sino una emancipación de su statu quo y una praxis en la cual enfermería se involucre con el cuidador, de manera que se produzcan transacciones beneficiosas para ambos.

Al comparar el Zarit y el Pulses, llama la atención que a pesar de que el enfermo o familiar tenga una dependencia alta, los cuidadores informan una media de sobrecarga moderada. Posiblemente, la cultura latina y otros factores influyen en sentirse moderadamente cargado. Se requiere un estudio cualitativo que despeje estas inquietudes.

\section{Conclusiones}

El estudio aporta datos de los cuidadores y sus familiares con enfermedad crónica o grado de dependencia, que servirán para el desarrollo de actividades educativas tanto en la universidad como en la Alcaldía de Medellín.

En nuestro medio, la mayoría son cuidadoras, solteras, encargadas del hogar y cuidan a un familiar (padrehijo). La sobrecarga del cuidado es moderada, a pesar de cuidar personas con dependencia severa.

El cuidado no debe ser responsabilidad de un solo miembro de la familia; ha de ser un trabajo compartido por varios miembros con verdaderas funciones de cuidado.

En necesario continuar con el apoyo de los profesionales de la salud, en especial de enfermería y de la Alcaldía de Medellín, para sobrellevar el cuidado que brindan los cuidadores de personas con enfermedad crónica o discapacidad.

Para la enfermería es necesario el desarrollo de su disciplina que permita brindar un cuidado basado en la evidencia.

Quisiéramos añadir que durante el desarrollo de la investigación no se presentaron limitaciones. 


\section{Referencias}

1. Medellín cómo vamos [internet]. Medellín [citado 2019 octubre 27]. Disponible en: http://www.medellincomov amos.org/la-ciudad/

2. Municipio de Medellín. Informe de calidad de vida de Medellín, 2017. En Medellín cómo vamos: salud [internet]. 2017 [citado 2017 octubre 29]. Disponible en: https://www.medellincomovamos.org/download/como-vamos -en-salud-informe-de-calidad-de-vida-de-medellin-2017/

3. Collazos JM, Álzate A, Pacheco R. Caracterización de la población con discapacidad en las cinco ciudades principales de Colombia. Boletín [internet]. 2018 jun [citado 2019 oct 29];(9). Disponible en: https://www.minsalud.gov .co/sites/rid/Lists/BibliotecaDigital/RIDE/DE/PS/boletin-9-discapacidad.pdf

4. Carreño-Moreno SP, Chaparro-Díaz L. Calidad de vida de los cuidadores de personas con enfermedad crónica. Aquichan. 2016;16(4):447-61. http://dx.doi.org/10.5294/aqui.2016.16.4.4

5. Acuerdo 27 de 2015, por medio del cual se establece una política pública para cuidadoras y cuidadores familiares y voluntarios de personas con dependencia de cuidado y conformación de Redes Barriales de Cuidado en el Municipio de Medellín. Gaceta OAcial 4340 [internet]. [Citado 2016 nov 9]. Disponible en: https://www.medellin.gov.co/irj/go/km/docs/pccdesign/SubportaldelCiudadano_2/PlandeDesarrollo_0 _15/Publicaciones/Shared\%20Content/GACETA\%20OFICIAL/2015/Gaceta\%204340/ACUERDO\% 200027\%20DE\#202015.pdf

6. Galvis-López CR, Aponte-Garzón LH, Pinzón-Rocha ML. Percepción de la calidad de vida de cuidadores de pacientes asistentes a un programa de crónicos, Villavicencio, Colombia. Aquichan. 2016;16(1):104-15. https: //doi.org/10.5294/aqui.2016.16.1.11

7. Bierhalsa C, Lowb G, Paskulinc L. Quality of life perceptions of family caregivers of older adult's stroke survivors: a longitudinal study. Appl Nurs Res. 2019;47:57-62. https://doi.org/10.1016/j.apnr.2019.05.003

8. Jeonga A, Shinb Jong D, Hyock Parkc J, Parkd K. Attributes of caregivers' quality of life: A perspective comparison between spousal and non-spousal caregivers of older patients with cáncer. J Geriatr Oncol. 2020;11(1):82-7. h ttps://doi.org/10.1016/j.jgo.2019.05.020

9. Siew-Tim L, Wan-Yen T, Chen-MunWo M, Kheng-Seang L, Sherrini Bazir A, Chong-Tin T. Burden in caregivers of adults with epilepsy in Asian families. Seizure. 2019;71:132-9. https://doi.org/10.1016/j.seizure.2019.07.008

10. Avilés-Cura MA, Morales-Ramírez M, Benavides-Ibarra MC, Reyna-Salazar LL, Riquelme-Heras H, RamírezAranda JM, et al. Impacto de la parálisis cerebral en la carga y funcionalidad familiar. Rev Med Hosp Gen Méx. 2014;77(2):53-7.

11. Zea MC, Torres BP. Adultos mayores dependientes hospitalizados: la transición del cuidado. Invest Educ Enferm. $2007 ;(25) 1: 40-9$.

12. Gómez MM, de la Cuesta Benjumea C. Impacto en la biografía de los cuidadores de pacientes con demencia [tesis de maestría] [internet]. Medellín: Universidad de Antioquia; 2004 [citado 2016 nov 15]. Disponible en: http:/ /tesis.udea.edu.co/bitstream/10495/158/1/ImpactoCuidadoresPacientesDemencia.pdf

13. Gómez Gómez, MM. Estar ahí, al cuidado de un paciente con demencia. Invest Educ Enferm [internet]. 2007;XXV(2):60-71. Disponible en: http://www.scielo.org.co/pdf/iee/v25n2/v25n2a06.pdf

14. Giraldo Molina CI, Franco Agudelo GM, Correa LS, Salazar MO, Tamayo AM. Cuidadores familiares de ancianos: quiénes son y cómo asumen este rol. Rev Fac Nac Salud Pública [internet]. 2005;23(2):7-14. Disponible en: ht tps://www.redalyc.org/articulo.oa?id=105215257006

15. Giraldo Molina CI, Franco Agudelo GM. Calidad de vida de los cuidadores familiares. Aquichan [internet]. 2006;6(1):38-53. Disponible en: https://aquichan.unisabana.edu.co/index.php/aquichan/article/view/79/16 3

16. De la cuesta Benjumea C. Cuidado artesanal la invención ante la adversidad. Medellín: Universidad de Antioquia; 2004.

17. Franco Agudelo GM. 10 años capacitando a los cuidadores familiares. Ponencia presentada en los 10 años de capacitación a cuidadores informales, Facultad de Enfermería, Universidad de Antioquia, Colombia. 
18. Alcaldía de Medellín, Observatorio de Envejecimiento y Vejez. Sala temática Amautta [internet]. [Citado 2019 nov 24]. Disponible en: https://www.medellin.gov.co/irj/go/km/docs/pccdesign/SubportaldelCiudadano_2/ PlandeDesarrollo_0_0/Shared\%20Content/Amautta/amautta/index.html

19. Hernández Sampieri R, Mendoza Torres CP. Metodología de la investigación. México DF: McGraw Hill; 2018.

20. Granger CV, Greer DS, Liset E, Coulombe J, O’Brien E. Measurements of outcomes of care for stroke patients. Stroke [internet]. 1975;6:34-41. Disponible en: http://stroke.ahajournals.org/content/6/1/34.full.pdf+htm

21. Zarit Burden Interview (ZBI) [internet] [citado 2016 dic]. Disponible en: https://eprovide.mapi-trust.org/instr uments/zarit-burden-interview

22. Resolución 8430 de 1993/4 de octubre, por la cual se establecen las normas cientíacas, técnicas y administrativas para la investigación en salud [internet]. [Citado 2019 oct 9]. Disponible en: https://www.minsalud.gov.co/sit es/rid/Lists/BibliotecaDigital/RIDE/DE/DIJ/RESOLUCION-8430-DE-1993.PDF

23. Consejo de las Organizaciones Internacionales de las Ciencias Médicas. Pautas éticas internacionales [internet]. [Citado 2016 sep 18]. Disponible en: http://www.bioetica.uchile.cl/pautas/pautas.htm

24. Coca SM, Ramos FO, Fernández R. calidad de vida en los cuidadores familiares de pacientes con enfermedad terminal, bajo un programa de cuidados paliativos domiciliarios. Rev Salud Pública. 2017;XXI(3):22-34. https ://doi.org/10.31052/1853.1180.v21.n3.17386

25. Sánchez RT, Molina EM, Gómez OR. Intervenciones de enfermería para disminuir la sobrecarga en cuidadores: un estudio piloto. Rev Cuid. 2016;7(1):1171-84. http://dx.doi.org/10.15649/cuidarte.y7il.25

26. Hanzeliková A, López-Muñoz F, Fusté Moreno R. PerAl sociodemográAco de los cuidadores de los pacientes geriátricos hospitalizados mayores de 75 años y su relación con la satisfacción. Enferm Global. 2017;16(2):375-88. https://doi.org/10.6018/eglobal.16.2.249861

27. Pascual Cuesta Y, Garzón Patterson M, Silva Williams H. Características sociodemográAcas de pacientes con enfermedad de Alzheimer y sus cuidadores principales. Rev Cubana Enferm [internet]. 2015 Jun [citado 2019 oct 26];31(2). Disponible en: http://www.revenfermeria.sld.cu/index.php/enf/article/view/319

28. Jiménez Ruiz I, Moya Nicolás M. La cuidadora familiar: sentimiento de obligación naturalizado de la mujer a la hora de cuidar. Enferm Glob. 2018;17(49):420-47. http://dx.doi.org/10.6018/eglobal.17.1.292331.

29. Mercado DA. Medellín, la ciudad del país que más está envejeciendo. El Tiempo [internet]. 2019 jul 27 [citado 2020 mar 20]. Disponible en: https://www.eltiempo.com/colombia/medellin/medellin-es-la-ciudad-del-pais-q ue-mas-esta-envejeciendo-394022

30. Gómez-Galindo AM, Peñas-Felizzola OL, Parra-Esquivel EI. Caracterización y condiciones de los cuidadores de personas con discapacidad severa en Bogotá. Rev Salud Pública. 2016;18(3):367-8. http://dx.doi.org/10.1544 6/rsap.v18n3.53048

31. Piratoba-Hernández BN, Rozo-Gutiérrez JN. Caracterización sociodemográAca del cuidador familiar de la persona mayor en una localidad de Bogotá-Colombia. MedUNAB 2015;18(1):51-7. https://doi.org/10.29375/01237 047.2189

32. Prieto-Miranda SE, Arias-Ponce N, Villanueva-Muñoz EY, Jiménez-Bernardino CA. Síndrome de sobrecarga del cuidador en familiares de pacientes geriátricos atendidos en un hospital de segundo nivel. Med Int Méx [internet]. 2015;31:660-8. Disponible en: https://www.medigraphic.com/pdfs/medintmex/mim-2015/mim156d.pdf

33. Ramón-Arbuésa E, Martínez-Abadíab B, Martín-Gómez S. Factores determinantes de la sobrecarga del cuidador: estudio de las diferencias de género [carta al editor]. Aten Prim. 2017;49(5):308-13. https://doi.org/10.1016/ j.aprim.2016.07.003

34. Blanco V, Guisandea MA, Sánchez MT, Otero P, López L, Lino Vázquez F. Síndrome de carga del cuidador y factores asociados en cuidadores familiares gallegos. Rev Esp Geriatr Gerontol. 2019;54(1):19-26. https://doi. org/10.1016/j.regg.2018.03.005

35. Pinzón EA, Carrillo GM. Carga del cuidado y calidad de vida en cuidadores familiares de personas con enfermedad respiratoria crónica. Rev Fac Nac Salud Pública [internet]. 2016;34(2):193-201. Disponible en: https://revista s.udea.edu.co/index.php/fnsp/article/view/21729 
36. Martínez Debs L, Lorenzo Ruiz A, Llantá Abreu MC. Carga del cuidador en cuidadores informales primarios de pacientes con cáncer de cabeza y cuello. Rev Haban Cienc Méd [internet]. 2018 [citado 2019 ago 25];18(1):126-37. Disponible en: http://www.revhabanera.sld.cu/index.php/rhab/article/view/2341.

37. Cantillo-Medina CP, Ramírez-Perdomo CA, Perdomo-Romero AY. Habilidad de cuidado en cuidadores familiares de personas con enfermedad crónica y sobrecarga percibida. Cienc Enferm. 2018;24(XX):164. https://doi.org $/ 10.4067 / s 0717-95532018000100216$

38. Sánchez Herrera B, Gallardo Solarte K, Montoya Restrepo LA, Rojas Martínez MV, Solano Aguilar S, Vargas LD. Carga Ananciera del cuidado familiar del enfermo crónico en la Región Andina de Colombia. Rev Cienc Salud [internet]. 2016 Dec [citado 2019 oct 27];14(3):339-50. Disponible en: http://www.scielo.o rg.co/scielo.php?script=sci_arttext\&pid=S1692-72732016000300003\&lng=en. http://dx.doi.org/10.12804/ revsalud14.03.2016.03

39. Torres-Pinto X, Carreño-Moreno S, Chaparro-Díaz L. Factores que influencian la habilidad y sobrecarga del cuidador familiar del enfermo crónico. Rev Univ Ind Santander Salud. 2017;49(2):330-8. https://doi.org/10.1 8273/revsal.v49n2-2017006

\section{Notas}

* Artículo de investigación. Este artículo es derivado de la investigación "La carga de cuidado de los cuidadores familiares inscrita en el sistema de información de la Universidad de Antioquia”. La Universidad de Antioquia Ananció las horas de las investigadoras.

\section{Licencia Creative Commons CC BY 4.0}

Cómo citar este artículo: Salazar Maya AM, Cardozo García Y, Escobar Ciro CL. Carga de cuidado de los cuidadores familiares y nivel de dependencia de su familiar. Investig Enferm Imagen Desarr. 2020;22. https ://doi.org/10.11144/Javeriana.ie22.cccf 